Guía para las mujeres y sus familias

\title{
Planificación del parto en una unidad liderada por matronas (ULM)
}

Introducción

Planificar donde dar a luz y criterios de admisión 


\section{Introducción}

El presente documento es la traducción de la Guía para usuarias sobre la atención al parto normal y los criterios de ingreso en una unidad de bajo riesgo de la Regulation and Quality Improvement Authority (RQIA) de Irlanda del Norte. RQIA es el organismo responsable de monitorizar e inspeccionar la disponibilidad y calidad de los servicios de salud en Irlanda del Norte, y alentar mejoras en la calidad de estos. Esta guía ha sido revisada metodológicamente, por pares. Y la traducción ha sido elaborada siguiendo las guías de traducción y adaptación de la Organización Mundial de la Salud.

A su vez, ésta ha sido revisada por usuarias, con la colaboración de la asociación Dona Llum, con el fin de evaluar su comprensión y posible adaptación en centros que forman parte de nuestro Sistema Nacional de Salud.

Las usuarias han considerado que es de fácil comprensión y que sería de utilidad añadir información sobre las opciones de manejo del dolor durante el parto en unidades de bajo riesgo.

En este documento cuando nos referimos a mujeres incluimos a hombres trans con capacidad gestante y personas no binarias. 


\section{Equipo}

\section{Coordinación}

Escuriet, Ramon. Matrón. Investigador principal del grupo de investigación GhenderS de la Universidad Ramon Llull (URL). Gerència de Processos Integrats de Salut, Servei Català de la Salut.
Martin-Arribas, Anna. Matrona. Doctoranda en Medicina por la Universidad Autónoma de Madrid y la EANS. Profesora en la URL. Miembro del grupo de investigación Ghenders.

\section{Grupo de trabajo}

Fernández Cuesta, Ana Isa- Palau, Roser. Matrona refe- Segura Lechuga, Maria. Mabel. Matrona. Licenciada en rente en Fundació Hospital trona en el Hospital de la Sanfilología inglesa. Matrona en Sant Joan de Déu de Marto- ta Creu i Sant Pau, Barcelona. ASSIR Lleida, Unidad Docen- rell, Barcelona. Doctoranda te de Matronas de Cataluña. Biomedicina Universitat PomVocal de matrona en el Colle- peu Fabra (UPF).

gi Oficial d'Infermeres i Infermers de Lleida. 


\section{Planificación del parto en una unidad liderada por matronas (ULM)}
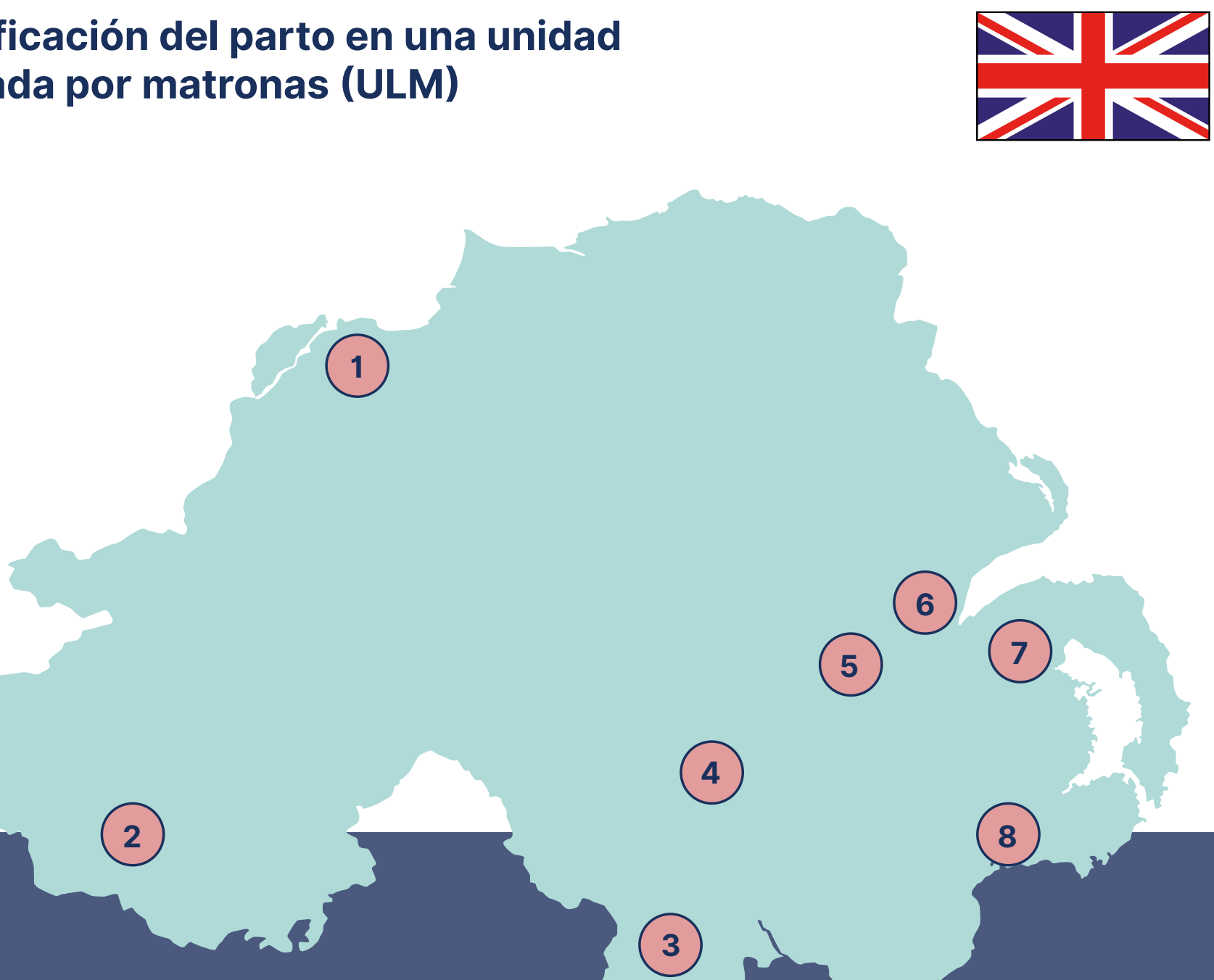

1. Altnagelvin ULPI abierta 2010

2. South Western Acute Hospital (SWAH) abierta 2013

3. Daisy Hill ULPI abierta 2014

4. Craigavon 1st ULPI abierta 2001

5. Lagan Valley ULPA abierta 2011

6. Mater ULPA abierta 2013

7. $\quad$ Ulster ULPI abierta 2008

8. Downe ULPA abierta 2010 


\section{¿Qué es una Unidad Liderada por Matronas (ULM)?}

Una ULM es una unidad de maternidad donde la matrona es el profesional de referencia y quien la dirige. En la actualidad existen ocho unidades en Irlanda del Norte.

- Cinco Unidades Integradas Lideradas por Matronas (UILM), ubicadas en el mismo complejo arquitectónico que las unidades obstétricas convencionales.

- Tres Unidades Autónomas Lideradas por Matronas (UALM), éstas se pueden encontrar a 15-40 minutos de una unidad obstétrica.

La evidencia científica del National Institute for Health and Care Excellence (NICE) pone de relieve que las unidades lideradas por matronas son especialmente adecuadas para mujeres sanas con un embarazo normal porque:

- Dar a luz a tu bebé en una ULM es tan seguro como en una unidad obstétrica convencional.

- En una ULM, tienes menores probabilidades de tener una cesárea y necesitar una transfusión sanguínea o un parto instrumentado para asistir el nacimiento de tu bebé.

\section{¿Dar a luz en una ULM?}

Las ULMs son especialmente adecuadas para mujeres sanas que tienen un embarazo normal. Un embarazo normal se define como aquel en que:

- No presentas ningún problema previo que afecte a este embarazo.

- Existen pocas probabilidades de que un problema de un embarazo o parto previo vuelva a ocurrir.

- En este embarazo no tienes ningún problema que requiera del seguimiento continuo de un médico obstetra

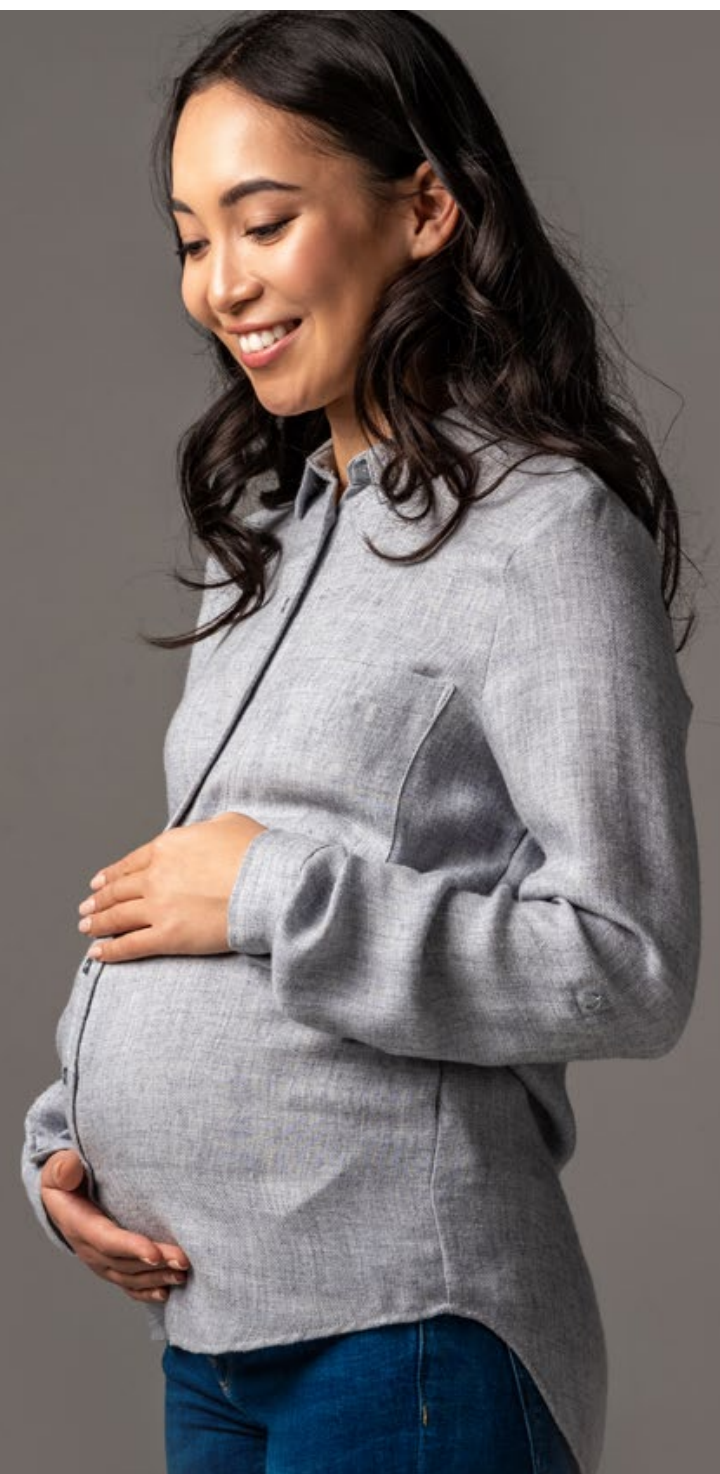




\section{Puedes planear dar a luz en cualquier ULM de Irlanda del Norte si:}

- Tienes entre 16 y 40 años en el momento de la primera visita.

- Tienes un Índice de Masa Corporal entre 18 y 35 en el momento de la primera visita.

- Tu última analítica presenta un valor de hemoglobina de al menos $100 \mathrm{~g} / \mathrm{l}$.

- No has tenido más de 4 partos previos.

- Este embarazo se ha conseguido mediante técnicas de reproducción asistida con citrato de clomifeno u otro tratamiento de fertilidad similar.

- Rompiste aguas de forma espontánea hace menos de 24 horas, no presentas signos de infección y te encuentras bien.

- Rompiste y son ligeramente verdosas pero, a parte de eso, te encuentras bien.

- Tienes o tuviste algún problema de salud mental que requiere atención de un profesional de la salud mental.

- Tuviste una amenaza de aborto, pero el embarazo ha continuado con normalidad.

- Tuviste una amenaza de parto prematuro y en la actualidad está resuelta.
- Inicialmente tu placenta estaba baja, pero ahora está localizada con normalidad.

- Dispones de una condición clínica que no afecta tu embarazo y tu salud general.

- Recibes apoyo de servicios sociales sin que afecte a tu embarazo o a tu salud.

- Tuviste un bebé con un problema de salud, pero en este embarazo no se ha detectado en tu bebé ninguna complicación.

- Tuviste un desgarro perineal de tercer grado que cicatrizó bien y no te ha provocado ninguna secuela.

- Tienes un resultado de una analítica sanguínea que muestra "anticuerpos séricos clínicamente no significativos sin relevancia clínica" (p.ej.: esto no tiene impacto en ti o en tu bebé).

- Tuviste algún tratamiento en el cérvix y has llegado a las 37 semanas de gestación sin ningún problema relacionado.

\section{Además, las mujeres que no cumplan los criterios descritos anteriormente, después de valorarlo y debatirlo, pueden optar por dar a luz en una UILM si:}

- Tienes menos de 16 años o más de 40 en el momento de la primera visita.

- Presentas un Índice de Masa Corporal $\geq 35$ e $\leq 40$ en la primera visita y dispones de buena movilidad.

- Tu última hemoglobina es de como mínimo $85 \mathrm{~g} / \mathrm{l}$ y ésta se reevaluará en el ingreso a la UILM.

- No has tenido más de 5 partos previamente.

- Has recibido un tratamiento de FIV, tu embarazo ha llegado a término (excepto si ha sido mediante ovodonación) y tu edad es inferior a 40 años. 
- Rompiste aguas de forma espontánea hace más de 24 horas, estás en fase de parto activo y no presentas signos de infección.

- Tienes o tuviste algún problema de salud mental que ha requerido tratamiento farmacológico, soporte adicional y ayuda de un profesional de salud mental.

- Tuviste una hemorragia postparto, pero no necesitaste una transfusión sanguínea o cirugía.

- Sufriste un desgarro vaginal o cervical extenso, o un desgarro perineal de tercer grado en un parto previo.

- Estás de parto debido a una inducción con prostaglandinas (tampón o gel, pero no perfusión de oxitocina).

- Eres portadora de Estreptococo Grupo B positivo (SGB) en este embarazo y no presentas signos de infección.

\section{¿Qué ocurre si me pongo de parto de forma prematura o postérmino?}

Se recomienda que des a luz en una ULM si tu embarazo se encuentra entre las 37 y las 42 semanas de gestación y cumples los criterios descritos en esta guía.

\section{¿Se aplicará esta guía en mi ULM de referencia?}

Estas directrices están implantadas en todas las unidades lideradas por matronas en Irlanda del Norte y se han desarrollado con el apoyo de la Red de Implementación de Guías y Auditorías (Guideline and Audit Implementation Network - GAIN - Ahora RQIA), el Departamento de Salud, Servicios Sociales y Seguridad Pública (DHSSPS) y los stakeholders clave de los servicios de maternidad.

Algunas mujeres, incluyendo las mujeres de mayor edad con un primer embarazo y las mujeres que sobrepasan en más de una semana su fecha prevista de parto, tienen una mayor probabilidad de necesitar un traslado a una unidad obstétrica convencional durante o inmediatamente después del parto.

Deberías buscar el asesoramiento de tu matrona de zona cuando desees planificar el lugar donde dar a luz. Si tienes alguna consulta o duda o dificultad, puedes concertar una cita con una matrona senior o hablar con tu matrona. 


\section{Información}

Podrá ver o imprimir una copia de este documento entrando en la página web de RQIA:

\section{www.rqia.org.uk}

Este recurso informativo y guía para las mujeres y sus compañeros deberá ser referenciada como:

RQIA (2016) "Planning to give birth in a midwife-led unit in Northern Ireland". RQIA: Belfast. www.raia.org.uk/RQIA/files/71/71cffe60-dd2d-405d-bd86-5728f5623fab.pdf

RQIA (GAIN 2016) "Guideline for Admission to Midwife-Led Units in Northern Ireland \& Northern Ireland Normal Labour \& Birth Care Pathway". RQIA: Belfast. www.rqia.org.uk/RQIA/files/3a/3a7a37bb-d601-4daf-a902-6b60e5fa58c2.pdf

\section{Referencias relacionadas:}

Healy, M. and Gillen, P. (2016) "Planning birth in, and admission to a midwife-led unit: development of a GAIN evidenced based guideline". Evidenced based Midwifery, 14(3), 82-86.

\section{Contacto}

Dra. Maria Healy. Chair, presidenta, directora del proyecto y autora principal. Profesora de Midwifery, Queen's University Belfast | maria. healy@qub.ac.uk (autora correspondiente).
Dra. Patricia Gillen. Co-directora del proyecto y autora principal. Jefa del R\&D para enfermeras, matronas y AHPs SHSCT/ Profesora Ulster University.

Contacto de referencia en España:

Anna Martin-Arribas.

annama7@blanquerna.url.edu 